\title{
Influence of surface morphology and structure of alginate microparticles on the bioactive agents release behavior
}

\author{
Slaven Jurića, Suzana Šegota ${ }^{\mathrm{b}}$, Marko Vincekovića,* \\ ${ }^{a}$ Department of Chemistry, University of Zagreb Faculty of Agriculture, Svetošimunska 25, 10000 Zagreb, Croatia \\ ${ }^{\mathrm{b}}$ Ruđer Bošković Institute, Laboratory for Biocolloids and Surface Chemistry, Bijenička c. 54, 10000 Zagreb, Croatia
}

\section{A R T I C L E I N F O}

\section{Keywords:}

Alginate microparticles

Biofertilizer

Release mechanism

structure/property relationship

Trichoderma viride

\begin{abstract}
A B S T R A C T
The structure-property relationship in alginate microparticles (microspheres and microcapsules prepared with or without Trichoderma viride spores (TV) was investigated. Surface morphology, structure and release behavior from alginate microparticles strongly depend on calcium concentration and presence of $T v$ and chitosan layer. All microparticles exhibited a granular surface structure with substructures consisting of abundant smaller particles. In vitro active agents release study revealed that the increase in calcium cation concentration reduced the release rate of $T v$ ( $\sim 84 \%$ for microspheres; $~ 57 \%$ for microcapsules) and calcium cations ( $20 \%$ for microspheres; $\sim 23 \%$ for microcapsules). The average decrease in $k$ values due to chitosan layer addition is $41 \%$ for $T v$ and $93 \%$ for calcium ions, respectively. The underlying $T v$ release mechanism from microspheres is anomalous transport kinetics, whereas from microcapsules is controlled by Type II transport. The differences in microparticle surface properties did not affect the mechanism controlling calcium ions release detected as diffusion through microparticles.
\end{abstract}

\section{Introduction}

The use of agrochemicals in agriculture had substantial repercussions for the environment, food security as well as human health because some of them are persistent organic pollutants. To diminish the overall exposure to agrochemicals, the worldwide intention is the restriction of their use and application of environmentally friendly formulations like biofertilizers and/or biopesticides. A biofertilizer is a substance which contains living microorganisms which, when applied to seeds, plant surfaces, or soil, colonize the rhizosphere or the interior of the plant and promotes growth by increasing the supply or availability of primary nutrients to the host plant (Vessey, 2003). Biofertilizer formulations usually contain a living microorganism (bacteria, fungi, nematodes) and a suitable carrier together with additives. Efficient formulation demands a carrier material which must preserve or maintain living organisms in a viable condition during storage and transport as well as must keep its functional properties after application. Microencapsulation is an advanced technology superior to prepare biofertilizer agroformulations in terms of protection living organism, improvement of their viability and possibility of controlled release into the field (John, Tyagi, Brar, Surampalli, \& Prévost, 2011).

Recently, we have shown the possibility of simultaneous encapsulation of copper (Vinceković et al., 2016; Vinceković, Jurić,
Đermić, \& Topolovec-Pintarić, 2017; Vinceković, Jurić et al., 2017) or calcium cations (Jurić, Đermić, Topolovec-Pintarić, Bedek, \& Vinceković, 2019) with Trichoderma viride spores (Tv) in biopolymers, alginate and chitosan, that easily create microparticles in which Tv can be incorporated using an aqueous system at ambient temperature. Trichoderma species are among the most prevalent culturable fungi in soils which antagonistic activity against plant pathogens and promotion of soil fertility make them as valuable biofertilizer. The physicochemical properties of calcium alginate microspheres loaded with $T$. viride spores and releasing behavior could be controlled by adjusting microsphere structure with a concentration of gelling cations (Vinceković et al., 2016; 2017). Numerous investigations have shown that coating of alginate microspheres with chitosan layer is and effective way of an active agent controlled release as a desirable characteristic for all delivery systems (Li et al., 2014; Lucinda-Silva, Salgado, \& Evangelista, 2010; Wang \& Zhao, 2013). Layering technology has been often utilized for producing novel materials with tailorable properties, such as, for example, multilayered chitosan/layered double hydroxides hybrid hydrogel as a promising controlled delivery system (Zhao et al., 2015, 2017).

The underlying hypothesis of our work is that improved understanding of the structure-property relationship in a biopolymer based agroformulation enhances the ability to control the release properties of

\footnotetext{
* Corresponding author.

E-mail addresses: sjuric@agr.hr (S. Jurić), ssegota@irb.hr (S. Šegota), mvincekovic@agr.hr (M. Vinceković).
} 
a biofertilizer and may aid in developing microparticles with specifically tailored properties. The aim of the present work was to investigate the effect of calcium cation concentration, and the presence of $T v$ and chitosan layer on the surface morphology and structure of alginate microparticles and the release behavior with the intention of delivering bioactive agents to plants at the rate that closely approximates plant demands over an extended period.

\section{Materials and methods}

\subsection{Materials}

Alginic acid sodium salt (CAS Number: 9005-38-3, M/G ratio of $\sim 1.56$, molecular weight 280000 ) $\mathrm{g} \mathrm{mol}^{-1}$ was purchased from Sigma Aldrich (USA). Medium molecular weight chitosan (CS) (CAS Number: 9012-76-4, 190,000-310,000 Da, Degree of deacetylation: Min 75\%) was obtained from Across Organic (USA). A commercially available product $\mathrm{CaCl}_{2}$ was purchased from Kemika (Croatia) and eosin, from Fluka (Buchs, Switzerland). All other chemicals were of analytical grade and used as received without further purification.

An indigenous isolate of Trichoderma viride (STP) originated from parasitized sclerotia of Sclerotinia sclerotiorum was used in all experiments (Topolovec-Pintarić, Žutić, \& Đermić, 2013). STP was grown for 10 days in potato dextrose broth (Biolife, Italy) under the constant aeration at $22^{\circ} \mathrm{C}$. Details of STP and spore suspension preparation were previously described (Vinceković et al., 2016).

\subsection{Microparticles preparation}

Both, microspheres (MS) and microcapsules (MC) are microparticles. A microsphere is a solid matrix particle, whereas a microcapsule has an inner core and outer shell. We have prepared MS in one step by ionic gelation, whereas MC was prepared in two steps by ionic gelation and polyelectrolyte complexation (Vinceković et al., 2016). Ionic gelation involves the preparation of MS by dripping a solution of sodium alginate without or with $T v$ into calcium chloride solution using Encapsulator Büchi-B390 (BÜCHI Labortechnik AG, Switzerland). The concentration of sodium alginate was constant $(1.5 \% \mathrm{w} / \mathrm{v})$, whereas initial calcium chloride concentrations varied from 0.5 to $2.0 \mathrm{~mol}$ $\mathrm{dm}^{-3}$. Microspheres (ALG/Ca or ALG/(Ca $\left.+T v\right)$ ) were formed in the cross-linking solution under mechanical stirring, then washed several times with sterilized water, filtered through Büchner funnel and stored at $4{ }^{\circ} \mathrm{C}$ until further studies.

Microcapsules were prepared by dispersing $50 \mathrm{~g}$ of washed microspheres in $100 \mathrm{ml}$ of chitosan solution $\left(0.5 \% \mathrm{CS}\right.$ in $\left.1.0 \% \mathrm{CH}_{3} \mathrm{COOH}\right)$ under constant stirring. Microcapsules without (CS/(ALG/Ca) or with $T v(\mathrm{CS} /(\mathrm{ALG} /(\mathrm{Ca}+\mathrm{T} v))$ were filtered, washed with deionized water and phosphate saline buffer, and stored at $4^{\circ} \mathrm{C}$ until further studies.

\subsection{Methods}

\subsubsection{Fourier transform infrared spectroscopy analysis}

The Fourier transform infrared spectroscopy (FTIR) spectra were recorded with the FTIR Instrument - Cary 660 FTIR (MIR system) spectrometer (Agilent Technologies, USA). Samples were mixed with potassium bromide to get pellets.

\subsubsection{Microscopic observations}

MS and MC size, morphology and topography were analyzed by several microscopic techniques: (i) optical microscopy (OM) (Leica MZ16a stereo-microscope, Leica Microsystems Ltd., Switzerland), (ii) scanning electron microscopy (SEM) (FE-SEM, model JSM-7000 F, Jeol Ltd., Japan)), (iii) confocal laser scanning microscopy (CLSM) (TCP SP2, Leica Lasertechnik, Germany) and (iv) atomic force microscopy (AFM) (Bruker Billerica, USA).

The average diameter of wet and dry microparticles was determined by optical microscopy using Olympus Soft Imaging Solutions GmbH, version E_LCmicro_09Okt2009. Twenty microparticles were randomly selected from batches produced in triplicate, to determine the size distribution.

CLSM was operated in transmitted and fluorescent mode at an acceleration voltage of $80 \mathrm{kV}$. All sample preparations were performed at room temperature. MC was stained with eosin $(0.01 \%)$ and placed on a microscope slide and sealed.

Microparticles for SEM analysis were put on the high-conductive graphite tape. FE-SEM was linked to an EDS/INCA 350 (energy dispersive X-ray analyzer) manufactured by Oxford Instruments Ltd. (UK). The ImageJ software was used for the determination the size of pores on a microparticle surface.

The samples for AFM imaging were prepared by deposition of a microparticle suspension on the mica substrate. The microparticles are flushed three times with $50 \mu \mathrm{l}$ of MiliQ water in order to remove all residual impurities. The microparticle surface, cross section and grain size distribution within each sample were analyzed using MultiMode Scanning Probe Microscope with Nanoscope IIIa controller (Bruker, Billerica, USA) with SJV-JV-130 V ("J" scanner with vertical engagement); Vertical engagement (JV) $125 \mu \mathrm{m}$ scanner (Bruker Instruments, Inc.); Tapping mode silicon tips (R-TESPA, Bruker, Nom. Freq. $300 \mathrm{kHz}$, Nom. spring constant of $40 \mathrm{~N} / \mathrm{m}$ ). In this manner, three-dimensional information about the surface topology was obtained and the roughness was quantified. All AFM imaging was performed at three different regions of each microparticle to ensure consistency of obtained results.

\subsubsection{Swelling degree and fraction of released bioactive agents}

Detailed procedures for the determination of swelling degree $\left(\mathrm{S}_{\mathrm{w}}\right)$ and a fraction of released $T v\left(\mathrm{f}_{T v}\right)$ and calcium ions $\left(\mathrm{f}_{\mathrm{Ca}}\right)$ from microparticles were previously described (Vinceković et al., 2016).

$S_{\mathrm{w}}$ was calculated using the equation:

$S_{w}=\frac{w_{t}-w_{0}}{w_{0}} \times 100$

where $w_{\mathrm{t}}$ is the weight of the swollen MS or MC, and $w_{0}$ is their initial weight.

In vitro release studies from microparticles were carried out at room temperature. Results are presented as the fraction of released $T v$ or calcium ions using the equation:

$f=\frac{R_{t}}{R_{t o t}}$

where $\mathrm{f}$ represents the fraction of $T v$ or calcium ions released, $\mathrm{R}_{\mathrm{t}}$ is the amount of $T v$ or calcium ions released at time $t$, and $R_{\text {tot }}$ is the total amount of $T v$ or calcium ions loaded in microparticles.

The concentration of $T v$ (expressed as the number of spores (NS) per $1 \mathrm{~g}$ of dry microparticles) was determined at $\lambda=550 \mathrm{~nm}$ and the concentration of calcium cations at $\lambda=650 \mathrm{~nm}$ using UV-VIS spectrophotometer (Shimadzu, UV-1700).

\subsection{Statistical analyses}

All experiments were carried out at the room temperature in triplicate for each formulation. The obtained data were analyzed with Microsoft Excel 2016 and XLSTAT statistical software add-in and IBM SPSS Statistics 22. The data are represented as mean values \pm standard deviation. Multivariate analysis (principal component analysis - PCA) was performed (Appendix B in Supplementary material).

\section{Results and discussion}

\subsection{Identification of intermolecular interactions in microparticles}

FTIR spectra of MS prepared at various calcium chloride concentrations revealed functional groups of all components interact with 


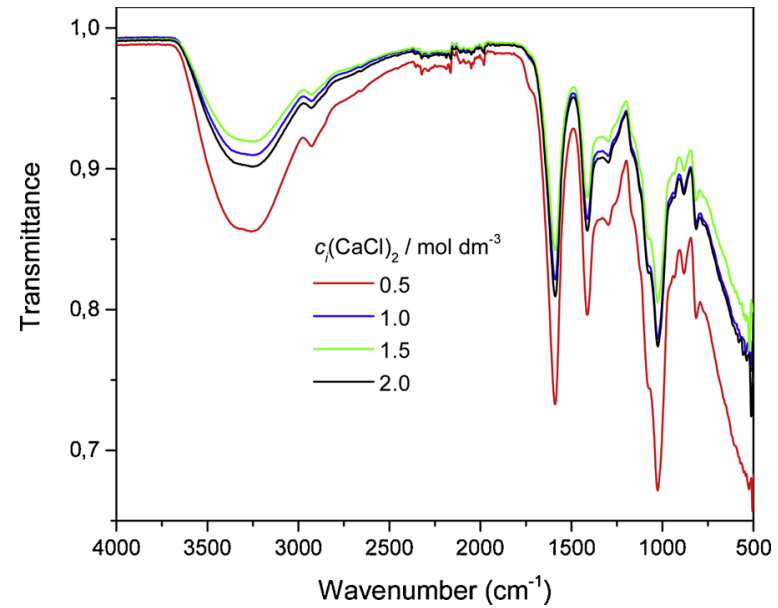

Fig. 1. FTIR spectra of $\mathrm{CS} /(\mathrm{ALG} /(\mathrm{Ca}+\mathrm{Tv}))$ microcapsules prepared at different initial calcium chloride concentration, $c_{i}\left(\mathrm{CaCl}_{2}\right) / \mathrm{mol} \mathrm{dm}^{-3}=0.5$ (red line), 1.0 (blue line), 1.5 (green line) and 2.0 (black line).

each other. Complex intermolecular interactions include mainly electrostatic interactions and hydrogen bonds. It was shown that the increase in calcium cation concentrations increases the crosslinking degree of calcium alginate microspheres, whereas the loading with $T v$ diminishes crosslinking degree due to the electrostatic repulsions between negatively charged $T v$ and free parts of alginate chains as well as mechanical interactions (Jurić et al., 2019).

FTIR spectra of MC prepared at various calcium chloride concentrations are given in Fig. 1. The strong and broad absorption band between 3500 and $3000 \mathrm{~cm}^{-1}$ recorded in all spectra belongs to the hydroxyl $(-\mathrm{OH})$ groups stretching vibrations and to the amine $\left(-\mathrm{NH}_{2}\right)$ groups superimposed on the hydroxyl groups due to the presence of chitosan coating layer. Besides bending vibration for amine groups superimposed on the hydroxyl, several characteristic peaks (at $1650 \mathrm{~cm}^{-1}$ for $-\mathrm{CO}$ stretching, at $1555 \mathrm{~cm}^{-1}$ for $-\mathrm{NH}_{2}$ bending vibrations, at $1370 \mathrm{~cm}^{-1}$ for asymmetric $-\mathrm{COC}$ and $-\mathrm{CN}$ stretching and $1150 \mathrm{~cm}^{-1}$ for $-\mathrm{CH}_{3}$ symmetrical deformation) disappeared or becomes weaker due to interaction between or superposition of the functional groups of chitosan and alginate (Sankalia, Mashru, Sankalia, \& Sutariya, 2007). The absence of chitosan band at $1582 \mathrm{~cm}^{-1}(-\mathrm{NH}$ bending vibration), reduced intensity and shifting of asymmetric $\left(1595 \mathrm{~cm}^{-1}\right)$ and symmetric $\left(1405 \mathrm{~cm}^{-1}\right)$ alginate carboxylate bands indicates electrostatic interactions between two oppositely charged polyelectrolytes (Vinceković et al., 2016).

An increase in calcium cations concentration causes the most significant changes in the alginate functional groups region as was previously shown for microspheres (Jurić et al., 2019). As the concentration of calcium ions increases the intensity of the absorption band around $3400 \mathrm{~cm}^{-1}$ decreases from 0.5 to $1.5 \mathrm{~mol} \mathrm{dm}^{-3}$ and then somewhat increases at $2 \mathrm{~mol} \mathrm{dm}^{-3}$. The difference in the intensity of absorption band between MS (Jurić et al., 2019) and MC indicates changes in the intensity of hydrogen bonds between oxygen atoms of Gresidues and the calcium in the egg-box structure. The decrease in the intensity of absorption band around $3400 \mathrm{~cm}^{-1}$ indicates the diaxially linked guluronic acid residues form smaller cavities which accommodate a lesser amount of water (Shi, He, Teh, Morsi, \& Goh, 2011; Roy, Bajapi, \& Bajapi, 2009).

In both, MS and MC, the sodium alginate asymmetric and symmetric carboxylate peaks became broader exhibiting gradual intensity increasing and shifting of carboxylate ions stretching vibrations (asymmetric to a lower and symmetric carboxylate vibrational peak to a higher wavenumbers) with increasing calcium cation concentrations. Changes in FTIR spectra of MS and MC clearly show the presence of chitosan layer differently influences on their structures with increasing

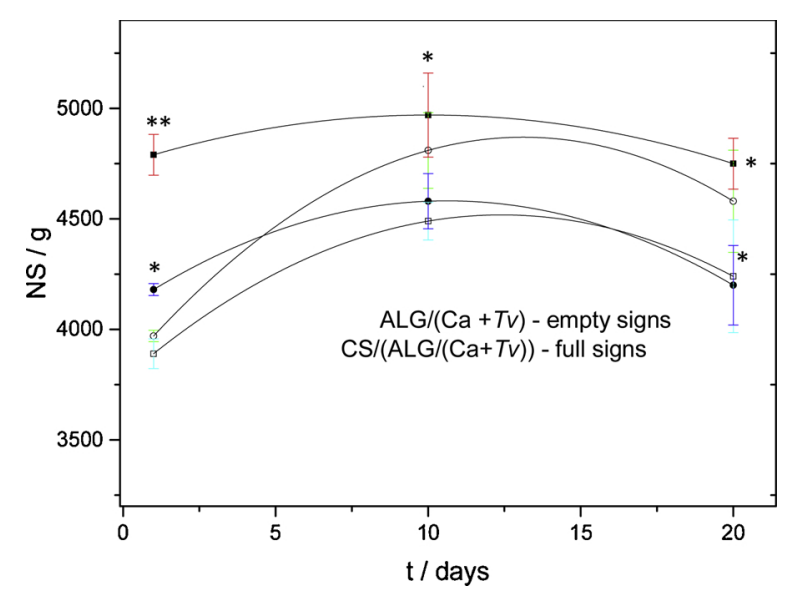

Fig. 2. Change in the number of $T v$ spores (NS/g) in $A L G /(C a+T v)$ microspheres (empty signs) (Jurić et al., 2019), and CS/(ALG/(Ca + Tv)) microcapsules (filled signs) prepared at initial calcium chloride concentration, $c_{i}\left(\mathrm{CaCl}_{2}\right) / \mathrm{mol} \mathrm{dm}^{-3}=1.0(\mathrm{O}, \mathrm{O}), 1.5(\square, \square)$ with time $(\mathrm{t})$. The error bars indicate the standard deviation of the means. * $(\mathrm{p}<0.05), * *(\mathrm{p}<0.001)$ statistically significant difference between MC and MS according to the posthoc Tukey HSD test.

calcium cation concentration.

\subsection{The viability of encapsulated Trichoderma viride spores in microparticles}

Our recent investigation has shown the interactions between calcium cations and negatively charged $T v$ stimulated germination during storage inside alginate microspheres (Jurić et al., 2019). Fig. 2. presents the change in the number of spores in microparticles with time. There was no significant change in NS between freshly loaded and 1 day stored in microparticles. During storage for 10 days, the NS increased by approximately $21 \%$ and $15 \%$ in MS (Jurić et al., 2019) and $10 \%$ and $7 \%$ in MC microparticles prepared at 1.0 and $1.5 \mathrm{~mol} \mathrm{dm}^{-3}$ of calcium chloride, respectively. During storage within 20 days, the NS somewhat decreased although remained still higher than in freshly prepared microparticles. The best biomass yield was observed in microparticles prepared at $1 \mathrm{~mol} \mathrm{dm}^{-3}$ of initial calcium chloride concentration. Experiments confirmed alginate microparticles prepared with calcium chloride provide an environment supportive of $T$. viride sporulation, although the presence of chitosan on the surface somewhat reduced the yield of spores.

\subsection{Size and morphology of microparticles}

Size, size distribution and shape optical microscopy observations revealed all prepared microcapsules were almost spherical, but after drying to constant mass (approximately four weeks on air at the room temperature) their sphericity was lost. The increase in calcium concentration from 0.5 to $2.0 \mathrm{~mol} \mathrm{dm}{ }^{-3}$ resulted in a decrease of MC size (approximately 16\%) as was also observed for MS (Jurić et al., 2019). This is in accordance with literature data showing the formation of smaller microparticles at higher $\mathrm{CaCl}_{2}$ concentrations due to a lower percentage of water retained and formation of more rigid gels (Daemi \& Barikani, 2012).

Wet MC prepared with $T v$ were slightly larger than those prepared without $T v$ implying change in the network structure due to the electrostatic repulsions between negatively charged $T v$ (Vinceković, Jurić et al., 2017) and free parts of alginate chains (the zeta potential of the calcium alginate matrix is about $-10 \mathrm{~V}$ ) (Rokstad, Lacik, de Vos, \& Strand, 2014) as well as mechanical interactions. The addition of Tv resulted in the size increase around 6, 10, 13 and 16\% in investigated calcium concentration range. As was observed for MS (Jurić et al., 


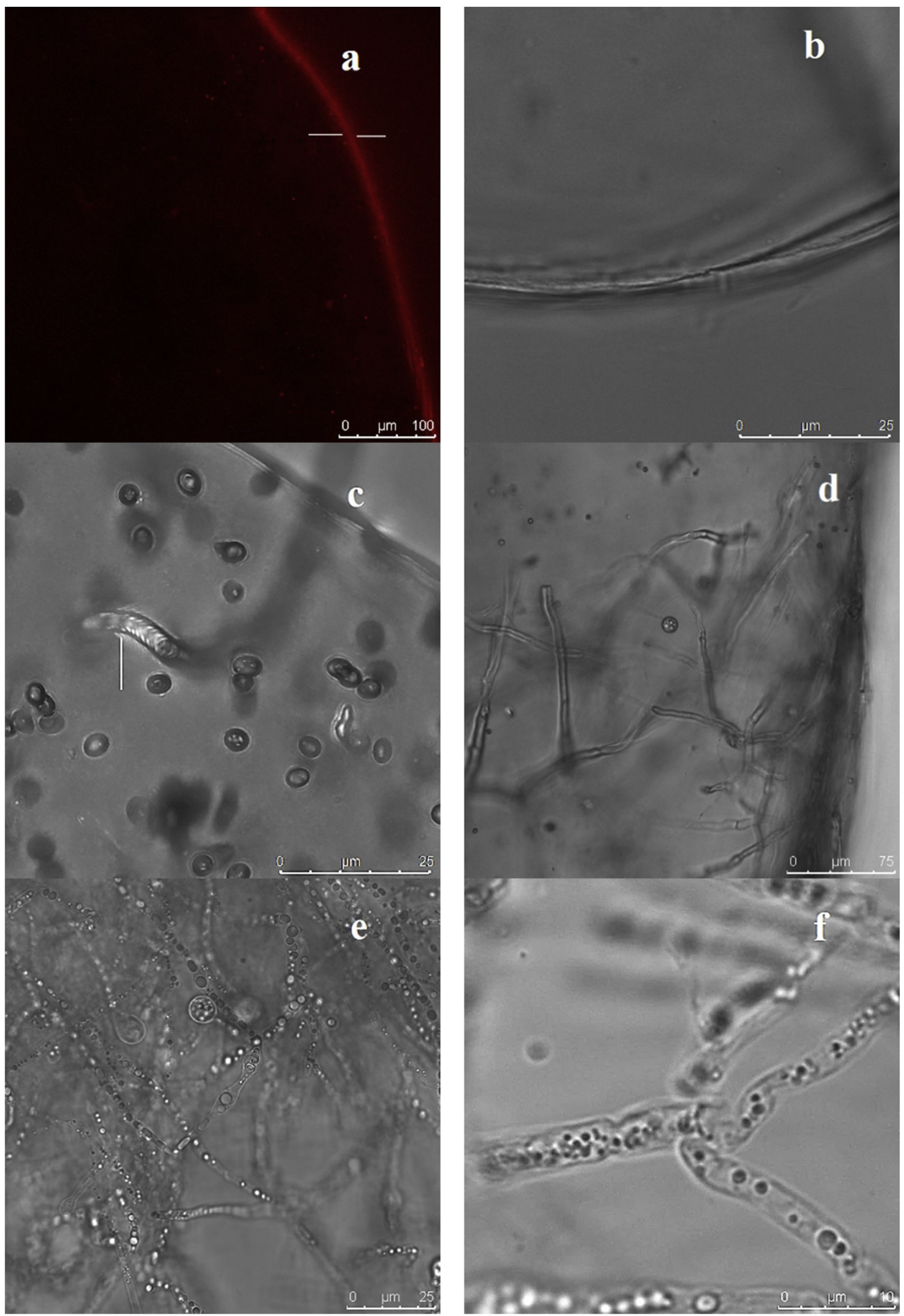

Fig. 3. CLSM microphotographs of (a) part of CS/(ALG/(Ca + Tv)) with chitosan layer (red, denoted by white lines) taken in fluorescence mode; (b) surface of $\mathrm{CS}$ / (ALG/Ca) and (c) $\mathrm{CS} /(\mathrm{ALG} /(\mathrm{Ca}+T v))$ with protruding germ tube, (d) sections of $\mathrm{CS} /(\mathrm{ALG} /(\mathrm{Ca}+T v)$ ) close to the surface, (e) branched hyphae growing inside $\mathrm{CS} /$ $(\mathrm{ALG} /(\mathrm{Ca}+T v)$ ), (f) enlarged picture of germ tubes inside $\mathrm{CS} /(\mathrm{ALG} /(\mathrm{Ca}+T v))$ taken in transmitted mode. Microcapsules were prepared at initial calcium chloride concentration, $c_{i}\left(\mathrm{CaCl}_{2}\right) / \mathrm{mol} \mathrm{dm}^{-3}=0.5$. Bars are indicated. 
2019), dried MC was also approximately two times smaller than the wet as a consequence of water and humidity loss associated with biopolymer strain-relaxation processes.

Microphotograph of a wet MC taken under CLSM in fluorescence mode (Fig. 3a) clearly shows the existence of thin chitosan layer on the surface. Chitosan layer became visible by staining with eosin, which due to its anionic character specifically binds to amino groups of chitosan. In contrast to MC prepared without $T v$ showing smooth surface (Fig. 3b), the surface of those prepared with Tv (Fig. 3c) exhibit numerous oval dimples (diameter around $3.6 \mu \mathrm{m}$ ) and a germ tube crossed the surface of microcapsule within a few days. Sections close to the surface revealed the presence of hyphae (Fig. 3d) and formation of mycelium inside the matrix (Fig. 3e). Enlarged microphotograph shows details of hyphae growing inside a microcapsule (Fig. 3f). All results clearly show encapsulated spores are able to germinate, that is calcium alginate microparticles with a cross-linked network containing a large fraction of water provide an environment supportive for Trichoderma viride germination. Presence of $T v$ and growth of mycelium inside the microparticle obviously change the gel network structure. The results are in accordance with investigations of De Jaeger, de la Providencia, Rouhier, and Declerck, (2011) who confirmed the ability of fungi to regrowth outside the calcium alginate coating and to colonize a susceptible plant.

The surface morphology of dried MS and MC observed by SEM are shown in Fig. 4a-f. The surface of MS prepared without Tv are highly porous with a pore size of approximately $0.169 \mu \mathrm{m}$ (Fig. 4a), whereas those prepared with Tv (Fig. 3b) exhibits numerous spherical blebs close to the size of individual spores ( $3.6 \mu \mathrm{m})$ (Jurić et al., 2019). Striped and fibrous surfaces representing the chitosan layer can be seen on MC surface (Fig. 4c). In comparison with MS, the MC surface porosity is reduced. Loading of MC with $T v$ resulted in a sleeker surface with the appearance of numerous dimples (Fig. 4d). Enlarged images (Fig. 4e,f) shows the structure of assembled holes from which the germ tubes penetrate out of the MC.

AFM analysis was performed using 2D- and 3D-topographic height images and topographic images of amplitude data (Fig. A1), and surface profiles taken along the white lines in images plotted in Fig. 5 (Fig. 5). The average size and mean diameter of grains on the surface and crosssection of microparticles and roughness are presented in Table 1. AFM analysis of a single (ALG/Ca) and (CS/ALG/Ca) prepared without $T v$ are given for comparison.

The surface area of the ALG/Ca is granular with clearly visible individual grains of height around $30 \mathrm{~nm}$. On the same surface, the topographic image of a larger resolution indicates the subordinate structure of an individual grain. The topographic image of the crosssection clearly shows the finer grain morphological characteristics. The grains are smaller with a height of $2-5 \mathrm{~nm}$ and have no subordinate structure.

Due to the presence of $T v$, the surface of $\mathrm{ALG} /(\mathrm{Ca}+T v)$ is smoother (roughness decreased from $R_{\mathrm{a}}=12 \pm 1 \mathrm{~nm}$ (ALG/Ca) to $R_{\mathrm{a}}=7.6 \pm 0.8 \mathrm{~nm}(\mathrm{ALG} /(\mathrm{Ca}+T v))$ and loses the grain structure forming a networked fine structure whose height of the cross-linked fibers reaches up to $40 \mathrm{~nm}$. On the same surface, the topographic image of a larger resolution indicates a subordinate structure within the network with a grain structure. The height of each individual grain reaches up to $10 \mathrm{~nm}$.

Images of a single $\mathrm{CS} /(\mathrm{ALG} / \mathrm{Ca})$ clearly shows a granular structure. The height of grains ranged from $30 \mathrm{~nm}$ to $100 \mathrm{~nm}$ showing a subordinate structure of an individual grain. The topographical image of the inner cross-section shows different morphological characteristics of the grains. They have no subordinate structure and are bigger than on the surface itself (average grain surface reached $122 \pm 143 \mathrm{~nm}$ ) and protrude above surface resulting in increased roughness of $R_{\mathrm{a}}=26 \pm 3 \mathrm{~nm}$.

Loading with $T v$ reduced the surface roughness from $R_{\mathrm{a}}=26 \pm 3 \mathrm{~nm}(\mathrm{CS} /(\mathrm{ALG} / \mathrm{Ca}))$ to $R_{\mathrm{a}}=16 \pm 3 \mathrm{~nm}(\mathrm{CS} /(\mathrm{ALG} /(\mathrm{Ca}$
$+T v)$ ). The enlarged surface of the $\mathrm{CS} /(\mathrm{ALG} /(\mathrm{Ca}+T v))$ revealed the presence of $T$, viride hyphae. Its presence does not affect the morphological characteristics of grains whose height is up to $5 \mathrm{~nm}$. Both microcapsules, with and without $T v$ have bigger grains (average grain surface reached $427 \pm 129 \mathrm{~nm}$ ), but grains are immersed in the matrix revealing in the decreased roughness value $R_{\mathrm{a}}=16 \pm 3 \mathrm{~nm}$.

The results confirmed that the microparticle surface morphologies are changing with the composition of the microparticle. A grainy surface texture with significant variation in microroughness suggests a porous morphology. The chitosan layer on microparticles increases surfaces roughness, but loading with $T v$ reduces the surface roughness of both, microspheres and microcapsules (Table 1).

\subsection{Swelling}

When microparticles were dispersed in water they swell. The increase in calcium concentration decreased the degree of swelling of all microparticles (Fig. 6) The concentration of calcium ions determines the kinetics of alginate gelation and the characteristics of the gel formed (Selimoglu \& Elib, 2010). During gelation, the carboxylate groups of guluronic acids react with calcium ions to form a crosslinked network of alginate chains. A higher $\mathrm{CaCl}_{2}$ concentration resulted in a higher crosslinking degree of network and a denser structure, which caused a less swelling when microparticles were dispersed in the solution. In comparison with MS, MC exhibited higher swelling degree due to the hydrophilic nature of chitosan-alginate complex on the surface.

\subsection{Mechanisms and kinetics of Trichoderma viride and calcium release from microparticles}

Design of controlled delivery systems involves the optimization of many parameters (preparation technique, microparticle chemical composition, geometry and size, the conditions during release etc.) among which are the most important type and concentrations of both, biopolymer and gelling cation (Irmanida, Devi, Kusdiantoro, \& Wahono Esthi, 2012). Having in mind all of the factors involved in the release and the combinatorial effect of different microparticle composition and the concentration of cross-linking cation (Rodrigues \& Lagoa, 2005) we have studied bioactive agents release from microparticles differing in surface morphology and structure.

It is known the most important rate-controlling release mechanisms from hydrophilic polymer microparticles are diffusion, swelling and erosion, that is the mechanisms and kinetics of release are determined by the rate of diffusion, swelling or erosion (Siepmann \& Siepmann, 2012). According to the Korsmeyer-Peppas model (Korsmeyer, Gurny, Doelker, Buri, \& Peppas, 1983) different controlling mechanisms may be distinguished by a simple empirical equation:

$f=k t^{n}$

where $k$ is a kinetic constant characteristic for particular system considering structural and geometrical aspects, $n$ is the release exponent representing the release mechanism, and $t$ is the release time. Values of $n<0.43$ indicates the release is controlled by classical Fickian diffusion, $n>0.85$ is controlled by Type II transport, involving polymer swelling and relaxation of the polymeric matrix. Values of $n$ between 0.43 and 0.85 show the anomalous transport kinetics determined by a combination of the two diffusion mechanisms and Type II transport.

Fig. 7a presents a comparison of Tv release profiles for MS (Jurić et al., 2019) and MC with increasing calcium cations concentration. All release profiles are characterized by rapid initial release (burst effect) and followed by slower release. They were analyzed by modified Eq. 3,

$f=a+k t^{n}$

where $a$ is the y-axis intercept characterizing the burst effect (Kim \& Fassihi, 1997; Lindner \& Lippold, 1995).

The fraction of released $T v$ from microspheres above 1 indicates a 

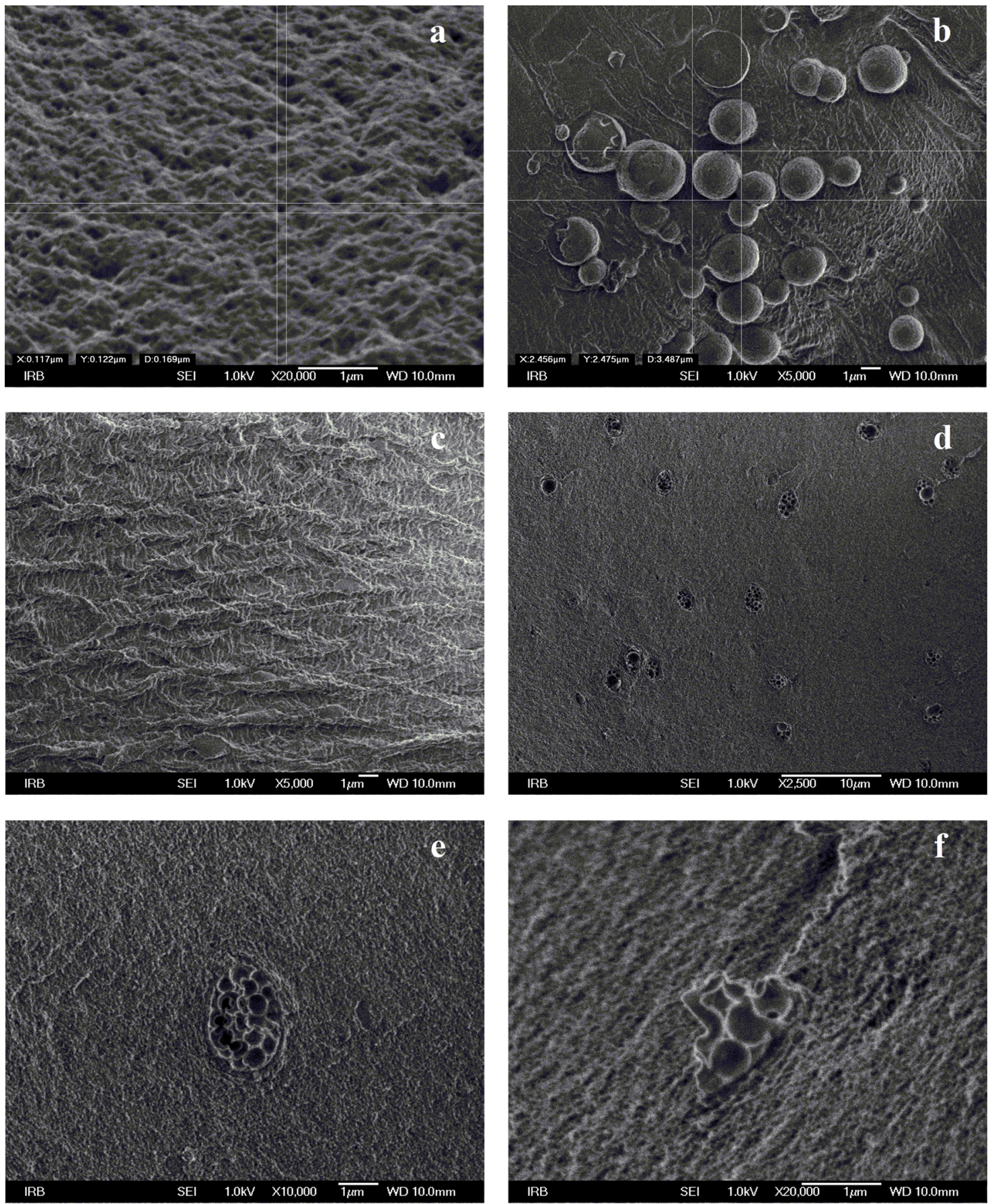

Fig. 4. SEM surface microphotographs of (a) ALG/Ca and (b) ALG/(Ca + Tv), (c) CS/(ALG/Ca) and (d,e,f) and CS/(ALG/(Ca + Tv)). Microparticles were prepared at initial calcium chloride concentration, $c_{i}\left(\mathrm{CaCl}_{2}\right) / \mathrm{mol} \mathrm{dm}^{-3}=0.5$. Bars are indicated.

higher concentration of Trichoderma viride biomass then loaded in the microsphere. The increasing amount of Trichoderma viride biomass in the surrounding medium is closely related to two sources, one is the release from microspheres and the other is germination. Microscopic observations confirmed such a conclusion revealing the growth of mycelium inside the microparticle matrix and germ tubes protruding toward the water phase. Coating of alginate microspheres with chitosan reduces the rate and amount of released $T v$ due to the additional barrier that slows transport from the MC to the surrounding solution.

The values of the intercept $a$, release constants $k$ and exponents $n$ are listed in Table 2. The average decrease in $k$ values (microspheres 84\%; microcapsules $~ 57 \%$ ) is in accordance with the effect of increasing gelling cation concentration on the strength of the alginate network structure. The average decrease in $k$ values due to chitosan layer addition is $\sim 41 \%$. Values of $n$ between 0.43 and 0.85 would point to the controlling release mechanisms from MS as anomalous transport kinetics (a combination of diffusion mechanisms and Type II transport). Obtained $n$ values close to or higher than 0.85 for MC indicate the release is controlled by Type II transport, that is by the polymer swelling and relaxation (transition of glassy structure to a rubbery state).

When calcium alginate microparticles are dispersed in water various physicochemical processes such as wetting, swelling (penetration of solution into the matrix) and polymer stress relaxation (transition of glassy structure to rubbery state), diffusion through the matrix, disintegration, dissolution or erosion of the structure, or their combination, may be included in the release of an active agent from a hydrophilic 


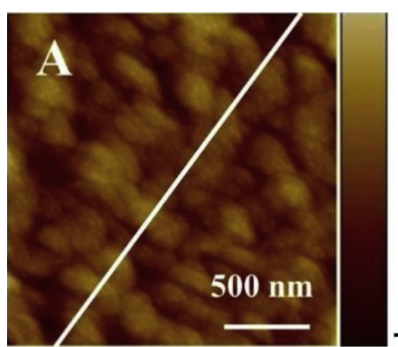

45 -
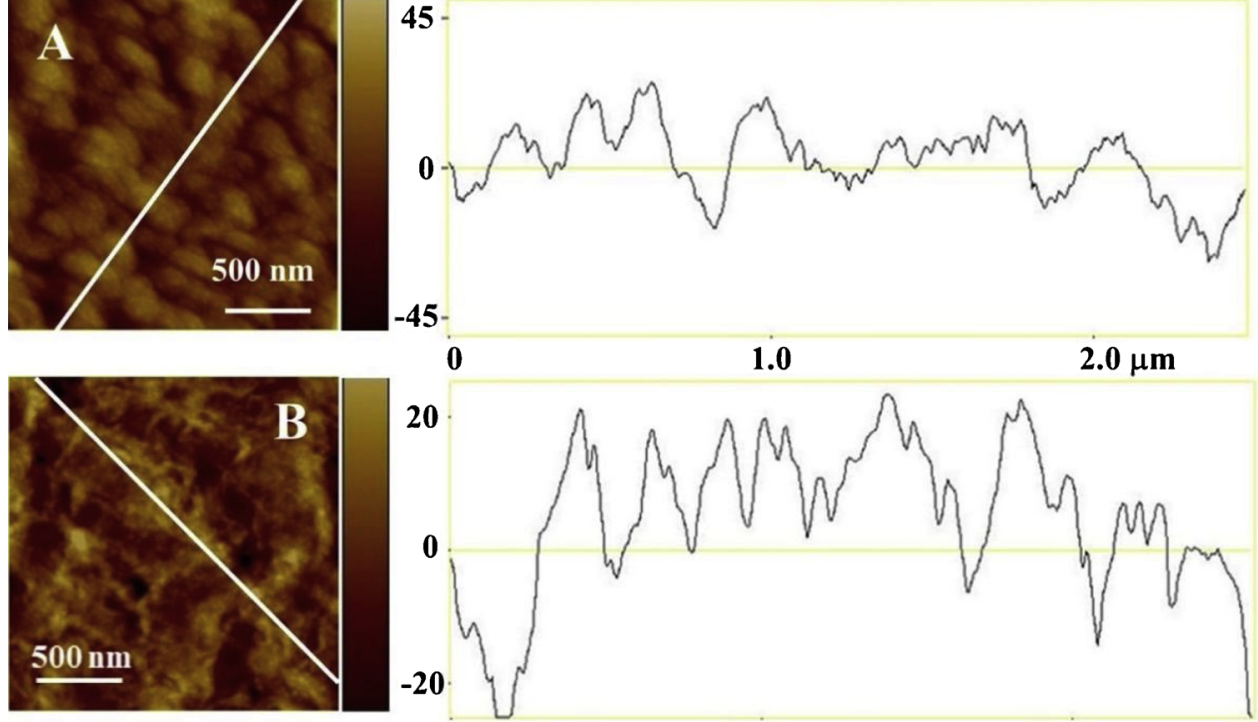

0

1.0

$2.0 \mu \mathrm{m}$
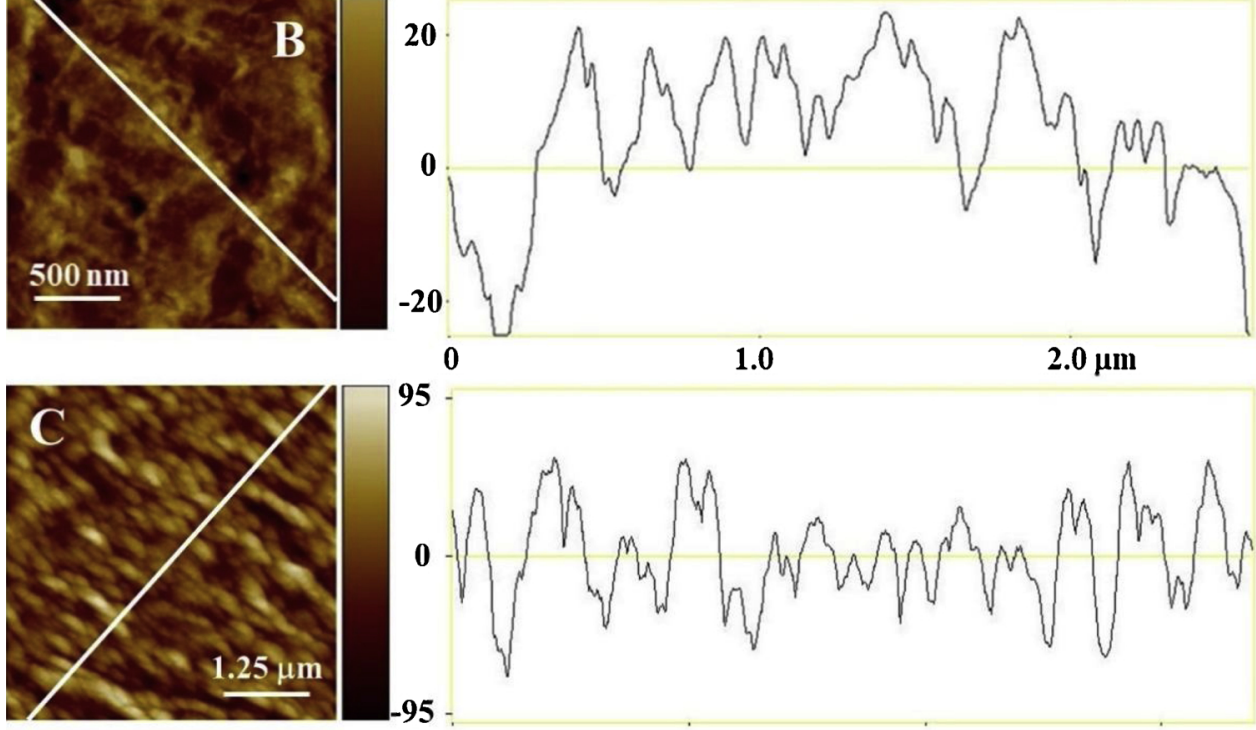

95.

$2.0 \mu \mathrm{m}$
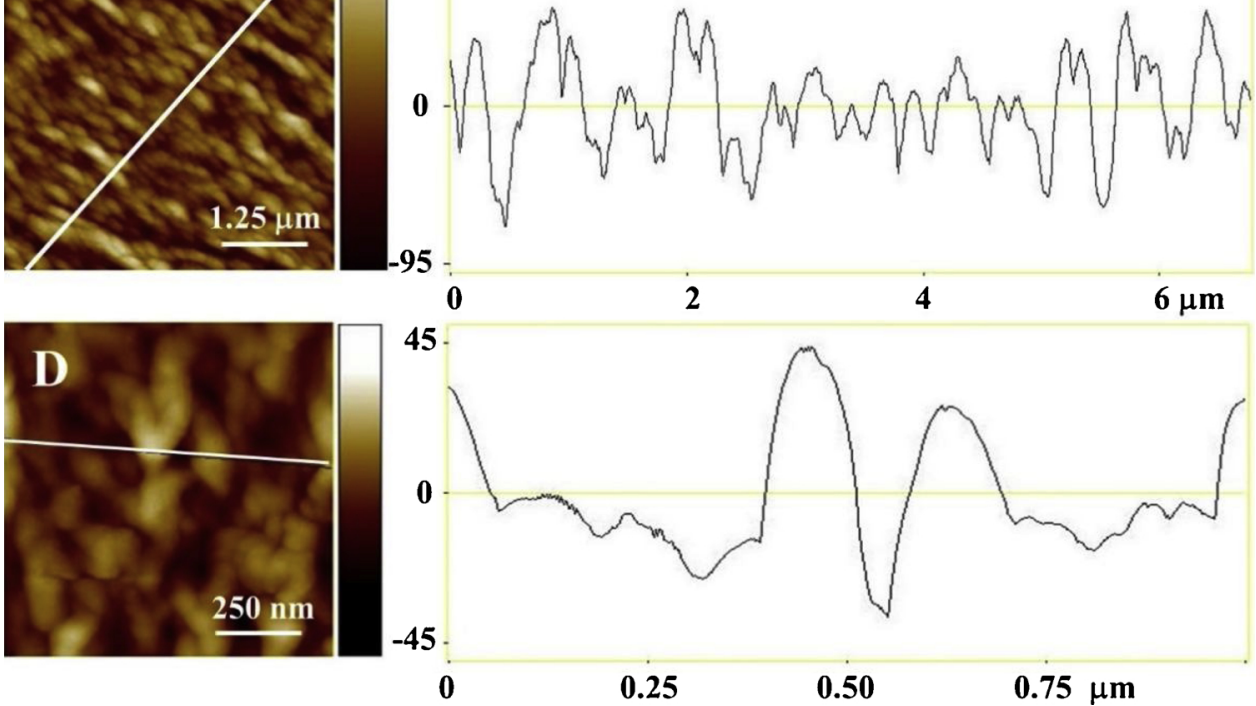

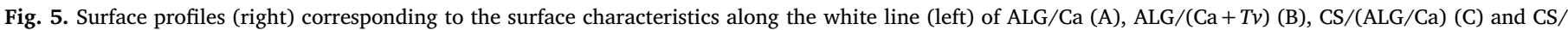
$(\mathrm{ALG} /(\mathrm{Ca}+T v))(\mathrm{D})$ microparticles. Microparticles were prepared at initial calcium chloride concentration, $c_{i}\left(\mathrm{CaCl}_{2}\right)=0.5 \mathrm{~mol} \mathrm{dm}-3$.

Table 1

Evaluated data of grain size (average and mean diameter) and surface roughness of microparticles: (a) surface and (b) cross-section prepared at initial calcium chloride concentration, $c_{i}\left(\mathrm{CaCl}_{2}\right) / \mathrm{mol} \mathrm{dm}^{-3}=0.5$.

\begin{tabular}{|c|c|c|c|c|}
\hline Microparticle & $\begin{array}{l}\text { Number of } \\
\text { grains }\end{array}$ & Average $/ \mathrm{nm}^{2}$ & $\begin{array}{l}\text { Mean } \\
\text { diameter/ } \\
\mathrm{nm}\end{array}$ & Roughness/nm \\
\hline \multicolumn{5}{|l|}{ (a) Surface } \\
\hline ALG/Ca & 80 & $33 \pm 20$ & $24 \pm 37$ & $12 \pm 1$ \\
\hline $\mathrm{ALG} /(\mathrm{Ca}+T v)$ & 159 & $79 \pm 110$ & $21 \pm 59$ & $7.6 \pm 0.8$ \\
\hline $\mathrm{CS} /(\mathrm{ALG} / \mathrm{Ca})$ & 89 & $122 \pm 143$ & $87 \pm 129$ & $26 \pm 3$ \\
\hline $\begin{array}{l}\mathrm{CS} /(\mathrm{ALG} /(\mathrm{Ca}+T v)) \\
\text { (b) } \underline{\text { Cross-section }}\end{array}$ & 18 & $427 \pm 129$ & $46 \pm 21$ & $16 \pm 3$ \\
\hline $\mathrm{ALG} / \mathrm{Ca}$ & 384 & $26 \pm 16$ & $24 \pm 47$ & $2.21 \pm 0.03$ \\
\hline $\mathrm{CS} /(\mathrm{ALG} / \mathrm{Ca})$ & 79 & $15 \pm 8$ & $64 \pm 27$ & $20 \pm 4$ \\
\hline
\end{tabular}

microparticle. Undoubtedly, all these processes somewhat collapse the structure of a microparticle and it is also expected the releasing of calcium ions. Calcium is an essential plant macroelement and promotes
$T v$ germination. Its release along with released $T v$ will have a beneficial impact on the plants, that is, they will act simultaneously.

The release of calcium cations from microparticles with an increasing concentration of calcium cations is presented in Fig. $7 \mathrm{~b}$. All release profiles are characterized by rapid initial release followed by slower release obeying a power law equation. To identify the kinetics and type of mechanism involved in the release Eq. 3 was applied. Values of $n<0.43$ indicate that the release process of calcium cations is controlled by diffusion through microparticles. In the measured time intervals both, MS and MC released a very small fraction of calcium. As is expected the amount and of released calcium ions from MC is smaller due to the presence of polyelectrolyte complex layer on the MC surface (Table 3).

The release of calcium cations from alginate microparticles prepared at the lowest calcium cation concentration is the fastest and decreases with increasing calcium cation concentration ( $k$ values decrease $\sim 20 \%$ for microspheres and $23 \%$ for microcapsules). This may be ascribed to the influence of calcium concentration on the gelation rate which controls microparticle homogeneity and percentage of retained 


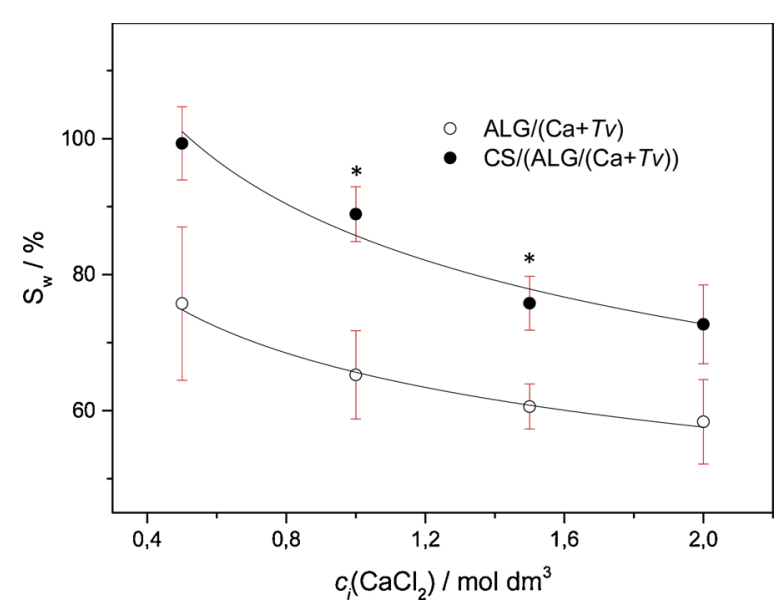

Fig. 6. Swelling degree $\left(S_{\mathrm{w}}\right)$ variation of microspheres $\mathrm{ALG} /(\mathrm{Ca}+T v)$ and microcapsules $\mathrm{CS} /(\mathrm{ALG} /(\mathrm{Ca}+T v))$ with initial calcium chloride concentrations, $c_{i}\left(\mathrm{CaCl}_{2}\right)$. The error bars indicate the standard deviation of the means. * ( $\mathrm{p}<0.05$ ), statistically significant difference between MC and MS according to the posthoc Tukey HSD test.
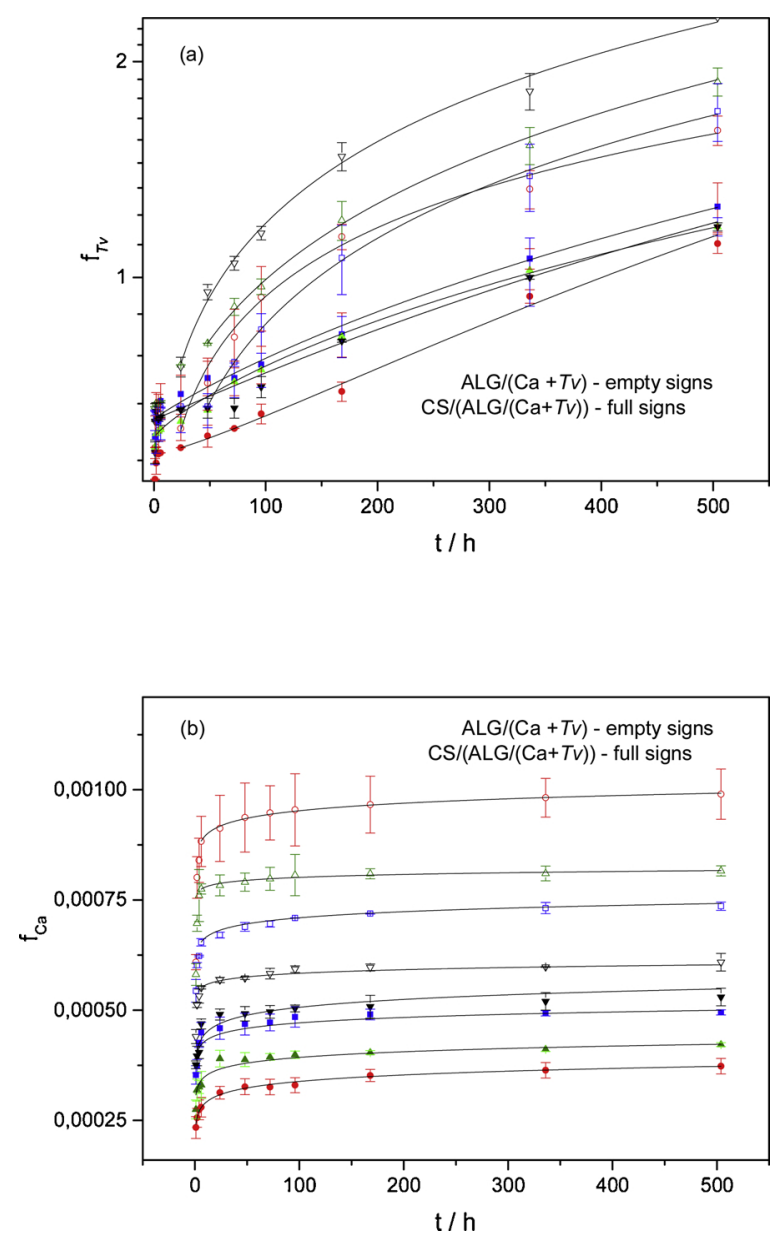

Fig. 7. (a) Fraction of released (a) $T v\left(\mathrm{f}_{T v}\right.$ ) and (b) calcium cations ( $\mathrm{f}_{\mathrm{Ca}}$ ) with time (t) from ALG/(Ca $+T v)$ microspheres (empty signs) and $\mathrm{CS} /(\mathrm{ALG} /(\mathrm{Ca}$ $+T v)$ ) microcapsules (full signs). Microparticles were prepared at initial calcium chloride concentration, $c_{i}\left(\mathrm{CaCl}_{2}\right) / \mathrm{mol} \mathrm{dm}^{-3}=0.5(\circ, \bullet), 1.0(\square, \square), 1.5(\Delta$, $\Delta)$ and $2.0(\nabla \nabla)$. The error bars indicate the standard deviation of the means (each sample had 3 replicates).

water (Rodrigues \& Lagoa, 2006). At lower cation concentration gelation is slower producing more uniform structures with a high
Table 2

Variation of the $y$-axis intercept $(a)$, the release constant $(k / h)$, exponent $(n)$, and correlation coefficient $\left(\mathrm{R}^{2}\right)$ of $T v$ released from $\mathrm{ALG} /(\mathrm{Ca}+T v)$ and $\mathrm{CS} /$ $(\mathrm{ALG} / \mathrm{Ca}+T v))$ at the initial calcium chloride concentration, $c_{i}\left(\mathrm{CaCl}_{2}\right) / \mathrm{mol}$ $\mathrm{dm}^{-3}$.

\begin{tabular}{lllll}
\hline$c_{i}\left(\mathrm{CaCl}_{2}\right)$ & $a$ & $k$ & $n$ & $\mathrm{R}^{2}$ \\
\hline $\mathrm{ALG} /(\mathrm{Ca}+\mathrm{Tv})^{*}$ & & & & \\
0.5 & 0.124 & 0.155 & 0.46 & 0.98 \\
1.0 & 0.227 & 0.059 & 0.52 & 0.99 \\
1.5 & 0.507 & 0.025 & 0.64 & 0.99 \\
2.0 & 0.550 & 0.025 & 0.70 & 0.99 \\
$\mathrm{CS} /(\mathrm{ALG} /(\mathrm{Ca}+\mathrm{Tv}))$ & & & & \\
0.5 & 0.541 & 0.0035 & 0.80 & 0.98 \\
1.0 & 0.597 & 0.0038 & 0.81 & 0.99 \\
1.5 & 0.612 & 0.0032 & 0.85 & 0.99 \\
2.0 & 0.620 & 0.0015 & 0.96 & 0.99 \\
\hline
\end{tabular}

* Jurić et al. (2019).

Table 3

Variation of the release constant $(k / h)$, exponent $(n)$ and correlation coefficient $\left(\mathrm{R}^{2}\right)$ of calcium released from $\mathrm{ALG} /(\mathrm{Ca}+T v)$ microspheres and $\mathrm{CS} /(\mathrm{ALG} / \mathrm{Ca}$ $+T v)$ ) microcapsules prepared at initial calcium chloride concentration, $c_{\mathrm{i}}\left(\mathrm{CaCl}_{2}\right) / \mathrm{mol} \mathrm{dm}^{-3}$.

\begin{tabular}{lllllll}
\hline$c_{\mathrm{i}}\left(\mathrm{CaCl}_{2}\right)$ & $k$ & $n$ & $\mathrm{R}^{2}$ & $k$ & $k$ & $\mathrm{R}^{2}$ \\
& $\mathrm{ALG} /(\mathrm{Ca}+T v)^{*}$ & & & $\mathrm{CS} /(\mathrm{ALG} /(\mathrm{Ca}+T v))$ & & \\
\hline 0.5 & $6.5 \times 10^{-4}$ & 0.07 & 0.99 & $3.1 \times 10^{-4}$ & 0.07 & 0.98 \\
1.0 & $6.1 \times 10^{-4}$ & 0.04 & 0.98 & $2.9 \times 10^{-4}$ & 0.04 & 0.98 \\
1.5 & $5.9 \times 10^{-4}$ & 0.03 & 0.99 & $2.5 \times 10^{-4}$ & 0.05 & 0.99 \\
2.0 & $5.2 \times 10^{-4}$ & 0.02 & 0.99 & $2.4 \times 10^{-4}$ & 0.06 & 0.98 \\
\hline
\end{tabular}

* Jurić et al. (2019).

percentage of water. The diffusion of calcium ions through brittle gels is faster than in stiffer gels formed at higher gelling cations concentration. The obtained differences in the kinetics can be attributed to the effects of calcium cations concentration on the microparticle structure as well as to the ability of calcium binding to Tv (Jurić et al., 2019). The presence of chitosan layer on the microcapsule surface further slows down the calcium cation release. The average decrease in $k$ values due to chitosan layer addition is $\sim 93 \%$.

\section{Conclusions}

The presented results contributed to a better understanding of the relationship between alginate microparticle structure and bioactive agents release properties. Calcium cation concentration, the presence of $T v$ and chitosan layer affect microparticle surface morphology and structure. Besides the effect of increasing calcium cation concentration, the presence of $T v$ and growth of mycelium inside the microparticles change the gel network structure. The surface of microparticles is granular with substructures consisting of abundant smaller particles. Microcapsules exhibited bigger grains and higher surface roughness than microspheres. Loading with $T$. viride reduced the surface roughness forming a sleeker surface with holes from which the germ tubes penetrated out of microparticles.

The mechanism of $T v$ release from microspheres is a combination of diffusion mechanism and the Type II transport (polymer swelling and relaxation). $T v$ release from microcapsules is controlled mainly by Type II transport indicating bigger surface grains on microcapsule surface slows down processes of the polymer swelling and relaxation. Differences in microparticle surface properties and structure do not affect the controlling mechanism of calcium cations release (detected as diffusion through microparticles) but influence the rate of calcium release. Both, the rates of $T v$ and calcium released from microcapsules are smaller due to the presence of the chitosan layer on the surface.

A better understanding of the relationship between structural 
properties of a microparticle and kinetics and mechanisms controlling the release of active agents enhances the ability to control their release behavior and may aid in developing new microparticles with tailored properties. Recently, we have applied variously designed microparticles simultaneously loaded with chemical and biological agents on several plants under greenhouse conditions and in an open field. After the treatments, grapevine vine leaves reached a significant increase in bioactive potential compared to the control (Vinceković et al., 2019). The results obtained on the other plants (lettuce, tomato, strawberries, tobacco) are very promising and their publication is under preparation.

\section{Author contributions}

The corresponding author designed the research work and wrote the manuscript. Other authors carried out work under the guidance of the corresponding author.

\section{Funding}

We acknowledge the financial support of the Croatian Science Foundation (Project: UIP-2014- 501 09-6462).

\section{Acknowledgments}

We are grateful to prof.dr. S. Topolovec-Pintarić and prof.dr. E. Dermić for Trichoderma viride spore's donation.

\section{Appendix A. Supplementary data}

Supplementary material related to this article can be found, in the online version, at doi:https://doi.org/10.1016/j.carbpol.2019.04.096.

\section{References}

Daemi, M., \& Barikani, M. (2012). Synthesis and characterization of calcium alginate nanoparticles, sodium homopolymannuronate salt and its calcium nanoparticles. Scientia Iranica, 19, 2023-2028.

De Jaeger, N., de la Providencia, I. E., Rouhier, H., \& Declerck, S. (2011). Co-entrapment of Trichoderma harzianum and Glomus sp. within alginate beads: Impact on the arbuscular mycorrhizal fungi life cycle. Journal of Applied Microbiology, 111, 125-135.

Irmanida, B., Devi, R., Kusdiantoro, M., \& Wahono Esthi, P. (2012). Leydig cells encapsulation with alginate-chitosan: Optimization of microcapsule formation. Journal of Encapsulation and Adsorption Sciences, 2, 15-20.

John, R. P., Tyagi, R. D., Brar, S. K., Surampalli, R. Y., \& Prévost, D. (2011). Bio encapsulation of microbial cells for targeted agricultural delivery. Critical Review of Biotechnology, 31, 211-226.

Jurić, S., Đermić, E., Topolovec-Pintarić, S., Bedek, M., \& Vinceković, M. (2019) Physicochemical properties and release characteristics of calcium alginate microspheres loaded with Trichoderma viride spores. Journal of Integrative Agriculture, 18(0), 2-16. https://doi.org/10.1016/S2095-3119(19)62634-1.

Kim, H., \& Fassihi, R. (1997). Application of a binary polymer system in drug release rate modulation. 2. Influence of formulation variables and hydrodynamic conditions on release kinetics. Journal of Pharmaceutical Sciences, 86, 323-328.

Korsmeyer, R. W., Gurny, R., Doelker, E., Buri, P., \& Peppas, N. A. (1983). Mechanisms of solute release from porous hydrophilic polymers. International Journal of Pharmaceutics, 15, 25-35.

Li, L., Wang, L., Li, J., Jiang, S., Wang, Y., Zhang, X., et al. (2014). Insights into the mechanisms of chitosan-anionic polymers-based matrix tablets for extended drug release. International Journal of Pharmaceutics, 476, 253-265.

Lindner, W. D., \& Lippold, B. C. (1995). Drug release from hydrocolloid embeddings with high or low susceptibility to hydrodynamic stress. Pharmaceutical Research, 12, 1781-1785.

Lucinda-Silva, R. M., Salgado, H. R. N., \& Evangelista, R. C. (2010). Alginate-chitosan systems: In vitro controlled release of triamcinolone and in vivo gastrointestinal transit. Carbohydrate Polymers, 81, 260-268.

Rodrigues, J. R., \& Lagoa, R. (2006). Copper cations ions binding in Cu-alginate gelation. Journal of Carbohydrate Chemistry, 25, 219-232.

Rokstad, A. M. A., Lacik, I, de Vos, P., \& Strand, B. L. (2014). Advances in biocompatibility and physico-chemical characterization of microspheres for cell encapsulation. Advanced Drug Delivery Reviews, 67-68, 111-130.

Roy, A., Bajapi, J., \& Bajapi, A. K. (2009). Development of calcium alginate-gelatin based microspheres for controlled release of endosulfan as a model pesticide. Indian Journal of Chemical Technology. 16, 388-395.

Sankalia, M. G., Mashru, R. C., Sankalia, J. M., \& Sutariya, V. B. (2007). Reversed chitosan-alginate polyelectrolyte complex for stability improvement of alpha-amylase: Optimization and physicochemical characterization. European Journal of Pharmaceutics and Biopharmaceutics, 65, 215-232.

Selimoglu, S. M., \& Elib, M. (2010). Alginate as an immobilization material for MAb production via encapsulated hybridoma cells. Critical Reviews in Biotechnology, 30, 145-159.

Shi, P., He, P., Teh, T. K. H., Morsi, Y. S., \& Goh, J. C. H. (2011). Parametric analysis of shape changes of alginate beads. Powder Technology, 211, 60-66.

Siepmann, J., \& Siepmann, F. (2012). Modeling of diffusion controlled drug delivery. Journal of Controlled Release, 161, 351-362.

Topolovec-Pintarić, S., Žutić, I., \& Đermić, E. (2013). Enhanced growth of cabbage and red beet by Trichoderma viride. Acta Agriculturae Slovenica, 101, 87-92.

Vessey, Y. K. (2003). Plant growth promoting rhizobacteria as biofertilizers. Plant and Soil, 255, 571-586.

Vinceković, M., Jalšenjak, N., Topolovec-Pintarić, S., Đermić, E., Bujan, M., \& Jurić, S. (2016). Encapsulation of biological and chemical agents for plant nutrition and protection: Chitosan/alginate microcapsules loaded with copper cations and Trichoderma viride. Journal of Agricultural and Food Chemistry, 64, 8073-8083.

Vinceković, M., Maslov Bandić, L., Jurić, S., Jalšenjak, N., Čaić, A., Živičnjak, I., et al. (2019). The enhancement of bioactive potential in Vitis vinifera leaves by application of microspheres loaded with biological and chemical agents. Journal of Plant Nutrition, 42, 543-558.

Vinceković, M., Jurić, S., Đermić, E., \& Topolovec-Pintarić, S. (2017). Kinetics and mechanisms of chemical and biological agents release from biopolymeric microcapsules. Journal of Agricultural and Food Chemistry, 65, 9608-9617.

Vinceković, M., Topolovec Pintarić, S., Jurić, S., Viskić, M., Jalšenjak, N., Bujan, M., et al. (2017). Release of Trichoderma viride spores from microcapsules simultaneously loaded with chemical and biological agents. Agriculturae Conspectus Scientificus, 82, 395-401.

Wang, H., \& Zhao, J. (2013). Encapsulation of the herbicide Picloram by using polyelectrolyte biopolymers as layer-by-layer materials. Journal of Agricultural and Food Chemistry, 61, 3789-3796.

Zhao, P., Liu, Y., Xiao, L., Deng, H., Du, Y., \& Shi, X. (2015). Electrochemical deposition to construct nature inspired multilayer chitosan/layered double hydroxides hybrid gel for stimuli responsive release of protein. Journal of Materials Chemistry B, 3, 7577-7584.

Zhao, P., Zhao, Y., Xiao, L., Deng, H., Du, Y., Chen, Y., et al. (2017). Electrodeposition to construct free-standing chitosan/layered doublehydroxides hydro-membrane for electrically triggered protein release. Colloids and Surfaces B: Biointerfaces, 158, 474-479. 